\title{
Molecular dynamics simulations of biomolecules
}

\author{
Martin Karplus and J. Andrew McCammon
}

Nature Struct. Biol. 9, 646-652 (2002).

The orientation of the molecule in Fig. $2 a$ was inconsistent with the description in the legend. The correct figure is reproduced here. In addition, the reference at the end of the Fig. 2 legend was incorrect. The correct reference should be ref. 48 . The correct sentence should read "(For more details, see http://mccammon.ucsd.edu/ and ref. 48)". The authors apologize for any inconvenience this may have caused.
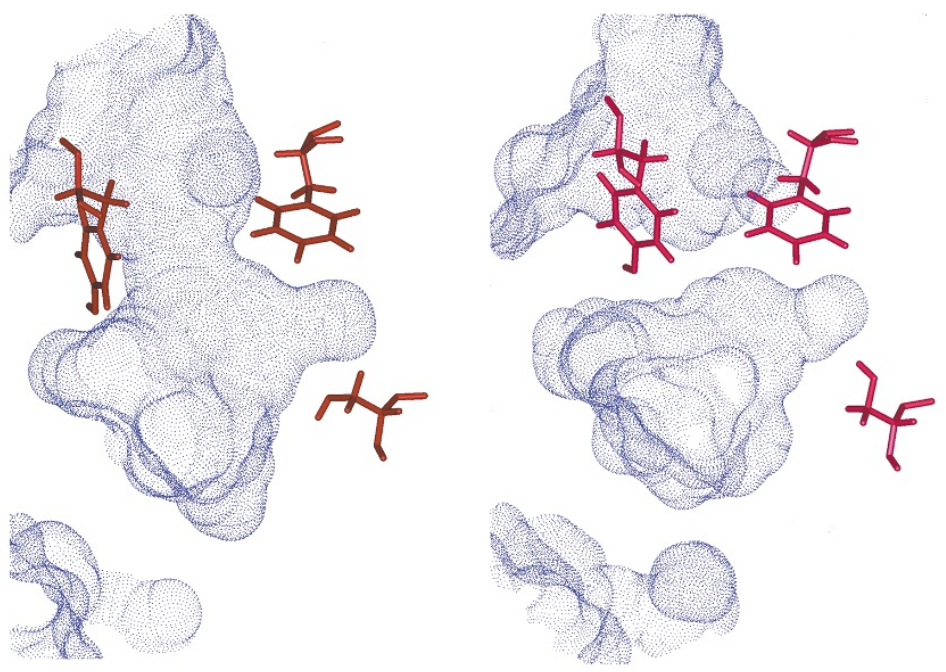

\section{Peroxisomal targeting signal-1 recognition by the TPR domains of human PEX5}

\author{
Gregory J. Gatto Jr., Brian V. Geisbrecht, Stephen J. Gould and Jeremy M. Berg \\ Nature Struct. Biol. 7, 1091-1095 (2000).
}

The sequence of the nonfunctional PTS1 variant peptide used in this study was reported as YQSEL. This is incorrect, and the correct sequence should be LQSEL. This mistake occurs at three places in the paper. The first is in the third sentence of the legend of Fig. 1. The correct sentence should read: "Titrations of PEX5-C into YQSKL (triangles) and LQSEL (squares) ...." The second is on page 1092, at the end of the first paragraph under the subheading "Overall structure of the complex". The correct sentence should read: “... a nonfunctional variant with Glu in place of Lys (LQSEL) ...”. The third is in the Methods section where "Tyr-Gln-Ser-Glu-Leu$\mathrm{COO}^{-}$(YQSEL)" should read "Leu-Gln-Ser-Glu-Leu-COO- (LQSEL)". It should be noted that the LQSEL peptide binds somewhat more tightly than does the YQSEL peptide. However, this difference does not affect the conclusions of the paper. The authors apologize for any inconvenience this mistake may have caused. 